\title{
Fighting back against resistant worms
}

Parasitic nematodes are an increasing threat to livestock and humans owing to the widespread development of resistance to the three major classes of anthelmintics: benzimidazoles, imidazothiazoles and macrocyclic lactones. Over the past 25 years only one new class has reached the market, the cyclodepsipeptides represented by emodepside (Bayer), but its use is restricted to cats so new drugs are urgently needed, particularly for livestock. Kaminsky and colleagues now report the discovery of the aminoacetonitrile derivatives (AADs) as a new class of anthelmintics.

To find promising candidates, over 600 AADs were synthesized and evaluated for anthelmintic activity. Those exhibiting high activity in an in vitro nematode larval development assay were then tested in a Mongolian gerbil model infected with the

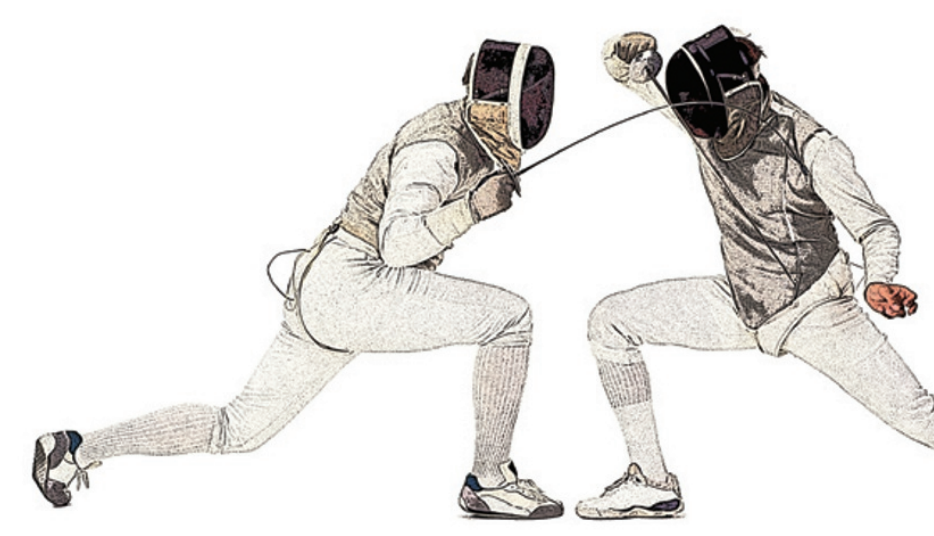

parasitic nematodes Haemonchus contortus or Trichostrongylus colubriformis. The most active compounds that were selected for further testing — AAD 1336 or 1470 - cured the infected gerbils, as well as sheep and cattle infected with various parasitic nematodes. The compounds also exhibited good pharmacokinetic profiles with slow elimination, good oral bioavailability and, as with all tested AADs, low toxicity and good tolerability in mammals.

Further studies showed that the AADs active against the parasitic nematodes tested had similar phenotypic effects on the movement, growth and viability of the nonparasitic nematode Caenorhabditis elegans. This gave the authors a well-studied model system to further investigate the mechanism of action of these AADs. Importantly, AAD 1470 was effective against ivermectin-, benzimidazole- and levamisoleresistant C. elegans, as well as against resistant strains in infected sheep, suggesting that the AADs have a novel mechanism of action.

To identify the AAD target, a genetic screen of AAD-resistant C. elegans was performed to find resistance alleles. Mutations in the gene $a c r-23$ were identified as a major contributor to AADresistance in C. elegans, which encodes the ACR-23 protein - a nematode-specific protein belonging to the DEG-3 group of nicotinic acetylcholine receptor subunits (nAChRs). Notably, the acr-23 mutants of C. elegans remained fully susceptible to other anthelmintic classes.

Next, to determine whether ACR-23 was also the target for AADs in parasitic nematodes, the authors probed for $\mathrm{nAChR}$ genes in AAD-resistant $H$. contortus mutants. Candidate genes were identified, sequenced and compared with sequence data from the ongoing $H$. contortus genome project and the completed C. elegans genome sequence. A gene with high similarity to C. elegans (des-2) was identified that, like $a c r-23$, encodes a protein that belongs to the DEG-3 group of nAChRs, suggesting that the nAChR pathway targeted by the AADs may be conserved between C. elegans and $H$. contortus. Identification of this pathway may help researchers to both design other AADs and devise strategies to slow development of resistance, if one member of this drug class is approved for use in the future.

Bethan Hughes

ORIGINAL RESEARCH PAPER Kaminsky, R. et al A new class of anthelmintics effective against drug-resistant nematodes. Nature 452, 176-180 (2008)

FURTHER READING Pink, R. et al. Opportunities and challenges in antiparasitic drug discovery. Nature Rev. Drug Discov. 4, 727-740 (2005) 